\title{
Omega-3 Polyunsaturated Fatty Acids Increase Plasma Adiponectin to Leptin Ratio in Stable Coronary Artery Disease
}

\author{
Magdalena Mostowik • Grzegorz Gajos • \\ Jaroslaw Zalewski • Jadwiga Nessler • Anetta Undas
}

Published online: 14 April 2013

(C) The Author(s) 2013. This article is published with open access at Springerlink.com

\begin{abstract}
Background Growing evidence suggests a cardioprotective role of omega-3 polyunsaturated fatty acids (PUFA). However, the exact mechanisms underlying the effects of omega-3 PUFA in humans have not yet been fully clarified.

Purpose We sought to evaluate omega-3 PUFA-mediated effects on adipokines in patients with stable coronary artery disease $(\mathrm{CAD})$ undergoing elective percutaneous coronary intervention (PCI).

Methods We conducted a prospective, double-blind, placebocontrolled, randomized study, in which adiponectin, leptin and resistin were determined at baseline, 3-5 days and 30 days during administration of omega-3 PUFA $1 \mathrm{~g}$ /day $(n=20)$ or placebo $(n=28)$.

Results As compared to controls administration of omega-3 PUFA resulted in increase of adiponectin by $13.4 \%(P<0.0001)$, reduction of leptin by $22 \%(P<0.0001)$ and increase of adiponectin to leptin (A/L) ratio by $45.5 \%(P<0.0001)$ at 30 days,
\end{abstract}

Magdalena Mostowik and Grzegorz Gajos contributed equally to this work.

Electronic supplementary material The online version of this article (doi:10.1007/s10557-013-6457-x) contains supplementary material, which is available to authorized users.

M. Mostowik $(\bowtie) \cdot$ G. Gajos $\cdot$ J. Zalewski $\cdot$ J. Nessler Department of Coronary Disease, Institute of Cardiology, Jagiellonian University Medical College, John Paul II Hospital, Pradnicka 80 st,

31-202 Krakow Poland

e-mail: magda.mostowik@gmail.com

A. Undas

Institute of Cardiology, Jagiellonian University Medical College, Krakow Poland

M. Mostowik · G. Gajos · J. Zalewski · J. Nessler · A. Undas John Paul II Hospital, Krakow Poland but not at 3-5 days. Compared with placebo adiponectin was $12.7 \%$ higher $(P=0.0042)$, leptin was $16.7 \%$ lower $(P<0.0001)$ and $\mathrm{A} / \mathrm{L}$ ratio was $33.3 \%$ higher $(P<0.0001)$ in the omega-3 PUFA group at 30 days. Resistin decreased similarly in both groups after 1 month, without intergroup differences $(P=0.32)$. The multivariate model showed that the independent predictors of changes in adiponectin at 1 month $(P<0.001)$ were: omega-3 PUFA treatment, baseline platelet count, total cholesterol and those in leptin $(P<0.0001)$ were: omega-3 PUFA treatment and waist circumference. Independent predictors of $\mathrm{A} / \mathrm{L}$ ratio changes $(P<0.0001)$ were: assigned treatment, current smoking and hyperlipidemia.

Conclusions In high risk stable coronary patients after PCI omega-3 PUFA supplementation improves adipokine profile in circulating blood. This might be a novel, favourable mechanism of omega-3 PUFA action.

Keywords Omega-3 polyunsaturated fatty acids .

Adiponectin $\cdot$ Leptin $\cdot$ Resistin $\cdot$ Stable coronary artery

disease $\cdot$ Percutaneous coronary intervention

\section{Introduction}

Growing evidence suggests a cardioprotective role of fish oil derived omega-3 polyunsaturated fatty acids (omega-3 PUFA), increased consumption of which has been shown to reduce the risk of coronary artery disease (CAD) [1]. It has been demonstrated that omega-3 PUFA may reduce plasma triglyceride (TG) level, arrhythmias, oxidative stress, inflammation, and improve endothelial dysfunction [1,2]. These agents can also produce antithrombotic effects including increased platelet response to clopidogrel [3], decreased thrombin formation, and favorable alterations to plasma fibrin clot properties [4]. However, the exact mechanisms underlying the effects of omega-3 PUFA in humans 
have not yet been fully clarified. In spite of this, current European prevention guidelines encourage consumption of PUFA in primary and secondary prevention of cardiovascular diseases [5]. However, recent randomized trials yielded inconsistent results showing that treatment with $1 \mathrm{~g}$ or less of omega-3 PUFA on top of guideline-adjusted therapy in high risk patients did not reduce the rate of major adverse cardiovascular events (MACE) [6].

Currently adipose tissue is perceived as an endocrine organ that produces bioactive peptides, adipokines, which exert multiple auto-, para- and endocrine effects [7]. Leptin, produced in adipocytes in response to changes in body fat, has been demonstrated to be an independent CAD risk factor [8]. Adiponectin is inversely proportional to visceral obesity and considered a cardioprotective cytokine through its insulin-sensitizing, antiatherogenic and antiinflammatory properties [9]. It has been indicated that hypoadiponectinemia could be an independent predictor of MACE after percutaneous coronary intervention (PCI) [9]. A role of resistin is less clear. It disturbs glucose and lipid metabolism, resulting in insulin resistance [10]. It has recently been reported that changes in adiponectin/ leptin $(\mathrm{A} / \mathrm{L})$ ratio were independent predictors of carotid intima-media thickness (IMT) alterations [11]. The potential of omega-3 PUFA to regress atherosclerosis has been shown in animal models [12]. Because omega-3 PUFA accumulate in adipose tissue [13], it may be hypothesized that some of their effects might be partly associated with altered fat tissue endocrine activity. Moreover, omega-3 PUFA have been shown to ameliorate low-grade inflammation in adipose tissue associated with obesity and to regulate adipokine gene expression [14]. A majority of the available studies carried out mostly in animals have provided inconsistent findings, showing beneficial, harmful or neutral effects of omega-3 PUFA on adipokine profile [15].

Little is known about omega-3 PUFA effects on adipokine levels and balance in high risk CAD patients, who potentially may benefit most from this therapy. The aim of the present study was to evaluate the effects of omega-3 PUFA on plasma adiponectin, leptin and resistin in high risk patients with stable CAD undergoing PCI.

\section{Methods}

We evaluated 48 patients, aged 30 to 80 , with stable CAD who underwent successful PCI with stent implantation enrolled in the OMEGA-PCI Study (Fig. 1), an investigatorinitiated, prospective, single-center, double-blind, placebocontrolled, randomized study. The complete study design has been published [3]. Exclusion criteria were as follows: acute coronary syndrome, severe obesity (defined as body mass

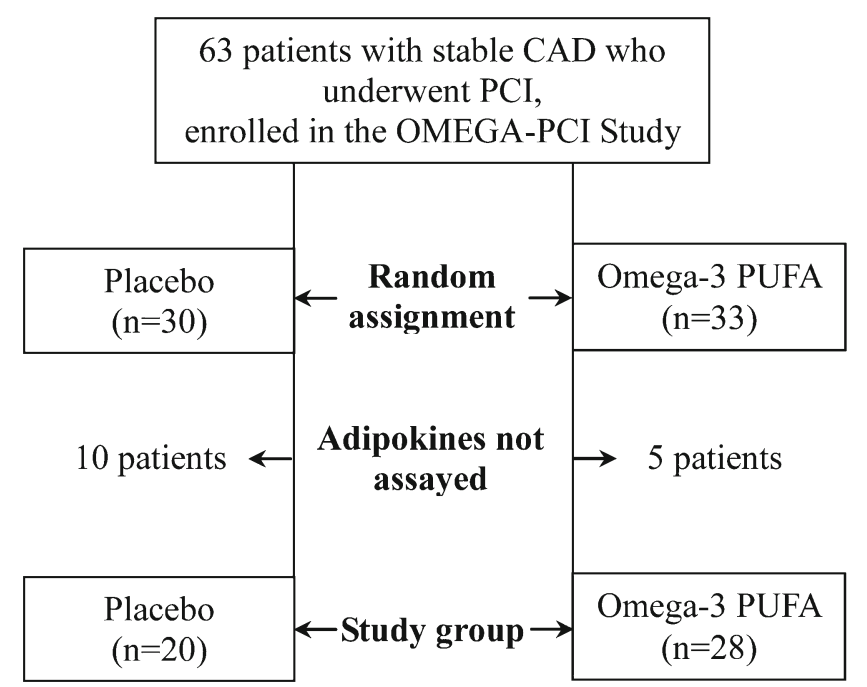

Fig. 1 Study flow chart. Abbreviations: CAD - coronary artery disease, $\mathrm{PCI}-$ percutaneous coronary intervention

index $[\mathrm{BMI}]>35 \mathrm{~kg} / \mathrm{m}^{2}$ ), history of inflammatory, autoimmune or infectious diseases, history of bleeding, oral anticoagulant therapy, thienopyridine use before enrolment, platelet count $<100,000 / \mu \mathrm{L}$, serum creatinine $>177 \mu \mathrm{mol} / \mathrm{L}(2 \mathrm{mg} / \mathrm{dl})$ or liver injury (alanine transaminase $>1.5$ times above the upper limit of the reference range). All patients denied taking fish oil supplements.

Metabolic syndrome was defined in accordance with the 2009 IDF statement [16] and hyperlipidemia as total cholesterol $>4.5 \mathrm{mmol} / \mathrm{l}$, low-density lipoprotein (LDL) cholesterol $>2.5 \mathrm{mmol} / \mathrm{l}$ or hypolipidemic treatment. Estimated glomerular filtration rate (eGFR) $<60 \mathrm{ml} / \mathrm{min}$ was considered as chronic kidney disease.

Briefly, before PCI, eligible patients on chronic acetylsalicylic acid (ASA) therapy were randomly assigned to receive clopidogrel (loading dose $600 \mathrm{mg}$ and thereafter $75 \mathrm{mg}$ once a day) and either omega-3 PUFA or placebo (soybean oil capsules) for 4 weeks. Omega-3 PUFA were administered in capsules containing ethyl esters of eicosapentaenoic acid $460 \mathrm{mg}$ and docosahexaenoic acid $380 \mathrm{mg}$. Compliance with medication was assessed based on the number of capsules returned at each visit in both groups. All patients were asked, according to the European Society of Cardiology recommendations, to increase consumption of oily fish. The assessment was performed at three time points: before study medication (I), at discharge from the hospital, 3-5 days after PCI (II) and 1 month after randomization (III). Ischemic and bleeding events were recorded during the trial.

The study was in compliance with the Good Clinical Practice International Conference on Harmonization rules and was approved by the Ethics Committee of the Jagiellonian University. Each patient provided written informed consent. 


\section{Laboratory Investigations}

Fasting blood samples were obtained between 8.00 and 10.00 AM, after at least $10 \mathrm{~h}$ of fasting, from an antecubital vein with minimal stasis. Routine blood tests, including lipid profile, blood cell count, glucose, serum creatinine and highsensitivity C-reactive protein, were assayed by automated laboratory techniques. Blood samples (vol/vol, 9:1 of $3.2 \%$ trisodium citrate) were centrifuged at $2500 \mathrm{~g}$ for $20 \mathrm{~min}$ within 30 min of collection and the supernatant was stored at $-80^{\circ} \mathrm{C}$. Adiponectin, leptin, and resistin were measured in plasma using the Bio Rad Luminex system (Millepore, Billerica, Massachusetts, United States), according to the protocol of the manufacturer. Coefficients of variation for the 3 analyses ranged from $6.5 \%$ to $10 \%$. All laboratory investigations were performed by the investigator blinded to the sample origin.

Statistical Analysis

Statistical analyses were performed with the Statistica 8.0 software package (Statsoft Inc.). Continuous variables were expressed as mean \pm SD or median (IQR) and categorical

Table 1 Baseline characteristics

$B M I$ body mass index, $M I$ myocardial infarction, $C A B G$ coronary artery bypass graft, $P C I$ percutaneous coronary intervention, $P A D$ peripheral artery disease, $C V D$ cardiovascular diseases, $C A D$ coronary artery disease, $A C E$ angiotensin converting enzyme, $A T 1$ angiotensin receptor 1

\begin{tabular}{|c|c|c|c|}
\hline & $\begin{array}{l}\text { Control } \\
N=20\end{array}$ & $\begin{array}{l}\text { PUFA } \\
N=28\end{array}$ & $P$-value \\
\hline Age (years) & $63.4 \pm 8.4$ & $62.5 \pm 10.0$ & 0.75 \\
\hline Female gender, $\mathrm{n}(\%)$ & $5(25)$ & $7(25)$ & 1.0 \\
\hline $\operatorname{BMI}\left(\mathrm{kg} / \mathrm{m}^{2}\right)$ & $28.5(25.5-33.0)$ & $29.0(25.5-31.5)$ & 0.82 \\
\hline Waist circumference $(\mathrm{cm})$ & $98.5(41.0-107.5)$ & $98.5(91.5-108.5)$ & 0.72 \\
\hline Previous MI, n(\%) & $10(50)$ & $7(25)$ & 0.074 \\
\hline Previous CABG, n(\%) & $1(5)$ & $4(14)$ & 0.30 \\
\hline Previous PCI, n(\%) & $5(25)$ & $3(11)$ & 0.19 \\
\hline Previous stroke or PAD, $\mathrm{n}(\%)$ & $7(35)$ & $7(25)$ & 0.45 \\
\hline \multicolumn{4}{|l|}{ CVD risk factors, $\mathrm{n}(\%)$ : } \\
\hline Hypertension & $19(95)$ & $27(94)$ & 0.81 \\
\hline Diabetes mellitus & $9(45)$ & $9(32)$ & 0.42 \\
\hline Hyperlipidemia & $20(100)$ & $27(94)$ & 0.39 \\
\hline Current smoking & $5(25)$ & $4(14)$ & 0.35 \\
\hline Family history of CAD & $11(55)$ & $17(61)$ & 0.69 \\
\hline Chronic kidney disease & $7(35)$ & $4(14)$ & 0.091 \\
\hline Metabolic syndrome & $14(70)$ & $19(68)$ & 0.87 \\
\hline Multivessel CAD & $15(75)$ & $21(75)$ & 1.0 \\
\hline \multicolumn{4}{|l|}{ Baseline laboratory investigations: } \\
\hline Creatinine $(\mu \mathrm{mol} / \mathrm{l})$ & $86.0(78.0-102.0)$ & $81.5(72.0-85.0)$ & 0.034 \\
\hline Hemoglobin $(\mathrm{g} / \mathrm{dl})$ & $14.1(13.5-15.2)$ & $14.3(13.2-14.6)$ & 0.81 \\
\hline White blood cell count $\left(\mathrm{x} 10^{3} / \mathrm{mm}^{3}\right)$ & $7.1 \pm 1.5$ & $7.4 \pm 2.0$ & 0.56 \\
\hline Platelet count $\left(\mathrm{x} 10^{3} / \mathrm{mm}^{3}\right)$ & $307 \pm 77$ & $325 \pm 87$ & 0.54 \\
\hline Fasting glucose (mmol/l) & $5.2(4.9-6.5)$ & $4.9(4.7-5.8)$ & 0.18 \\
\hline C-reactive protein (mg/l) & $2.1(1.1--2.4)$ & $2.2(0.9-2.6)$ & 0.57 \\
\hline Total Cholesterol (mmol/1) & $4.70(4.31-5.30)$ & $4.75(3.60-5.05)$ & 0.28 \\
\hline Low-density lipoproteins (mmol/1) & $3.00(2.35-3.35)$ & $2.60(2.10-3.05)$ & 0.13 \\
\hline High-density lipoproteins (mmol/l) & $1.05(0.90-1.20)$ & $1.00(0.96-1.10)$ & 0.99 \\
\hline Triglicerydes (mmol/1) & $1.90(1.20-2.40)$ & $1.59(1.00-1.70)$ & 0.07 \\
\hline \multicolumn{4}{|l|}{ Treatment, $\mathrm{n}(\%)$ : } \\
\hline Beta blocker & $15(75)$ & $25(89)$ & 0.19 \\
\hline ACE inhibitor & $18(90)$ & $24(86)$ & 0.66 \\
\hline AT1 antagonist & $1(5)$ & $7(25)$ & 0.067 \\
\hline Statin & $17(85)$ & $27(94)$ & 0.16 \\
\hline Fibrate & $1(5)$ & 0 & 0.23 \\
\hline Nitrate long acting & $10(50)$ & $11(39)$ & 0.46 \\
\hline Calcium antagonist & $12(60)$ & $11(39)$ & 0.16 \\
\hline
\end{tabular}


variables as numbers (percentage). Continuous variables were first checked for normal distribution by the Shapiro-Wilk statistic and then compared by Student $t$-test or the Mann-Whitney $U$ test, when appropriate. ANOVA for repeated measures assuming sphericity, with both contrast analysis for intergroup difference at each time point and post hoc analysis with Bonferroni correction for intragroup changes between different time points, was used for comparison of adiponectin, leptin and resistin. The chi-square test was used to evaluate the differences in categorical variables between the groups. The Pearson or Spearman rank correlation coefficient was calculated to test the association between two variables with a normal or non-normal distribution, respectively. All variables that showed the association with follow-up changes of adiponectin concentration or $\mathrm{A} / \mathrm{L}$ ratio in univariate model $(P<0.2)$ and did not show substantial correlations $(r>0.5)$ with another independent variable were then included in the multiple linear regression analysis to determine independent predictors. All statistics were 2 -sided. A probability value $<0.05$ was considered statistically significant.

The study was powered to have a $90 \%$ chance of detecting a $20 \%$ difference in adiponectin following omega-3 administration using a probability value of 0.05 , based on the values of adiponectin in CAD patients in a published paper [17]. To demonstrate such a difference or greater, at least 20 patients were required in each group.

\section{Results}

Forty-eight patients (36 men and 12 women; mean age $62.9 \pm 9.15$ years) were analysed. Baseline demographics, clinical characteristics and laboratory results of the subjects are shown in Table 1 .

There were no differences between the omega-3 PUFA and control groups except for creatinine at baseline which was slightly lower in the former group $(P=0.034)$. No intergroup differences were observed in adiponectin, leptin, resistin and $\mathrm{A} / \mathrm{L}$ ratio at baseline (Figs. 2 and 3).

During the study period there were only mild adverse events and their frequency did not differ between the groups. No MACE were noticed during the one-month follow-up in either group. There were no changes in concomitant medications throughout the trial.

\section{Effects of Omega-3 PUFA}

During the treatment period adiponectin increased by 13 . $4 \%$ in the omega-3 PUFA group as compared with controls $(F=12.5, \mathrm{df}=2, P<0.0001)$. After one-month follow-up adiponectin was $12.7 \%$ higher in subjects who received omega-3 PUFA as compared to those on placebo $(P=0$. 0042) (Fig. 2a).
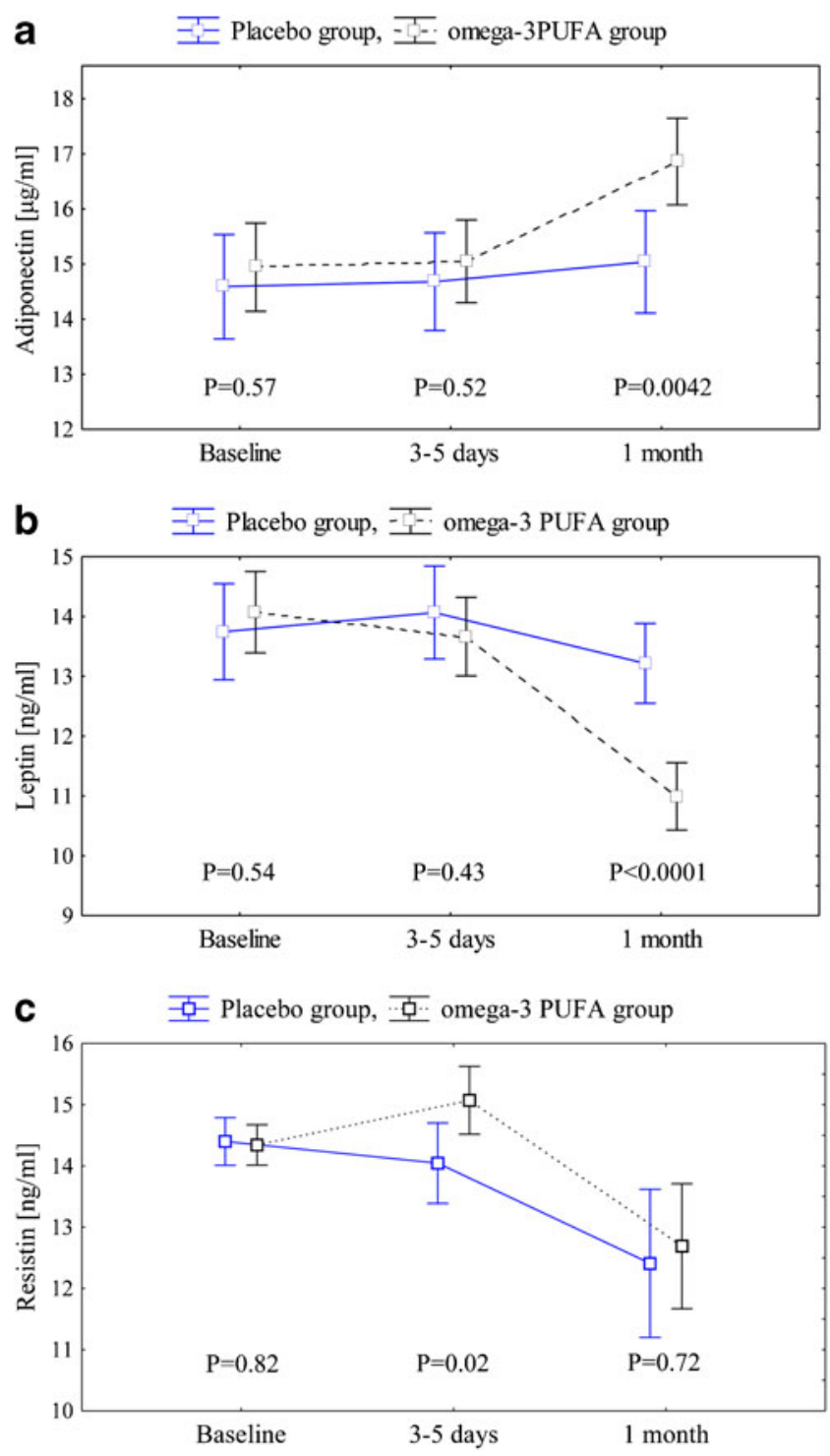

Fig. 2 a Adiponectin in the omega-3 PUFA and placebo groups. Abbreviations: data are shown as mean $\pm 95 \%$ CI. In the omega-3 PUFA group $P<0.001$ for both 1 month vs 3-5 days and 1 months vs baseline. No statistical significance for the placebo group. $F=12.5$, $\mathrm{df}=2, P<0.0001$. b Leptin in the omega-3 PUFA and placebo groups. Abbreviations: data are shown as mean $\pm 95 \%$ CI. In the omega-3 PUFA group $P<0.001$ for both 1 month vs 3-5 days and 1 month vs baseline. No statistical significance for the placebo group. $F=14.4, \mathrm{df}=$ 2, $P<0.0001$. c Resistin in the omega-3 PUFA and placebo groups. Abbreviations: data are shown as mean $\pm 95 \%$ CI. In the omega-3 PUFA group $P<0.001$ for 1 month vs baseline and $P=0.011$ for 1 month vs $3-5$ days, in the placebo group $P=0.009$ for 1 month vs baseline and $P=0.065$ for 1 month vs $3-5$ days. $F=1.15, \mathrm{df}=2, \mathrm{P}=0.32$

Leptin decreased during the treatment period by $22 \%$ in the omega-3 PUFA group as compared with controls $(F=14.4$, $\mathrm{df}=2, P<0.0001)$. After 1 month of treatment with omega-3 PUFA leptin was $16.7 \%$ lower than in the placebo group $(P<0.0001)$ (Fig. 2b).

During the treatment period $\mathrm{A} / \mathrm{L}$ ratio increased by $45.5 \%$ in the omega-3 PUFA group as compared with controls $(F=24.9$, 


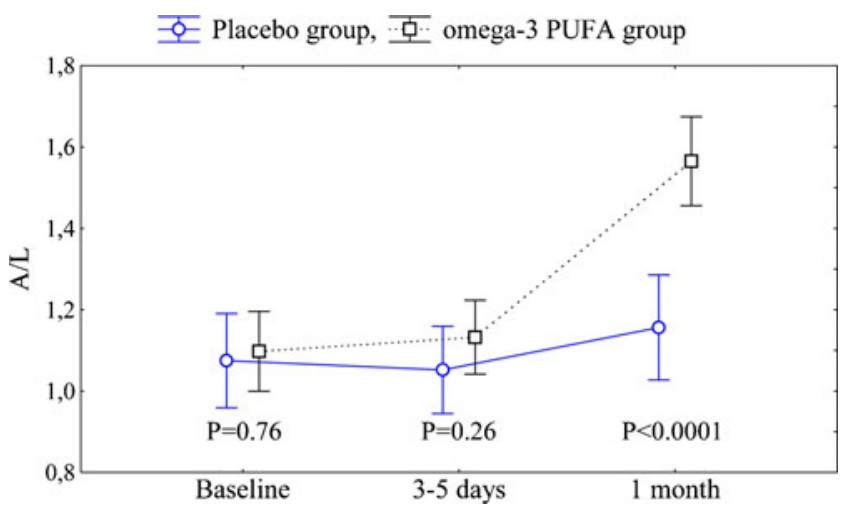

Fig. 3 Adiponectin-to-leptin ratio (A/L) in both groups. Abbreviations: data are shown as mean $\pm 95 \%$ CI. In the omega-3 PUFA group $P<0.001$ for both: 1 month vs 3-5 days and 1 month vs baseline. No statistical significance for the placebo group. $F=24.9$, df=2, $P<0.0001$

$\mathrm{df}=2, P<0.0001)$. After 1 month, A/L ratio was $33.3 \%$ higher in subjects who received omega-3 PUFA $(P<0.0001)$ (Fig. 3).

No changes in adiponectin or leptin were observed in the placebo group.

After 1 month resistin decreased by $11.2 \%(P<0.001)$ and by $13.9 \%(P=0.009)$ in the omega-3 PUFA and placebo groups, respectively, without intergroup differences throughout the study ( $F=1.15, \mathrm{df}=2, P=0.32$ ) (Fig. $2 \mathrm{c}$ ).

\section{Correlations}

Univariate linear regression showed no correlations between either BMI or waist circumference and adipokines in most of the time points. Current smoking positively correlated with leptin at baseline $(P=0.014)$ and at each time point in the placebo group $(P=0.002$ after $3-5$ days, $P=0.041$ after 1 month). There was an inverse correlation between fasting glucose and adiponectin at baseline $(P<0.001)$ and at each time point in the omega-3 PUFA group $(P=0.043$ after 3-5 days, $P=0.027$ after 1 month) as well as in controls ( $P=0.008$ after $3-5$ days, $P=0.004$ after 1 month); fasting glucose also positively correlated with leptin at baseline $(P=0.036)$. Total cholesterol positively correlated with resistin, but not with other adipokines, at baseline $(P=0.048)$ and after 1 month in the omega-3 PUFA group $(P=0.017)$ (Table A, B, C-supplementary material).

The multivariate model showed that the independent predictors of changes in adiponectin $(\mathrm{R} 2=0.62, P<0.001)$ were: treatment assignment to omega-3 PUFA, platelet count, total cholesterol 1 month after randomization and in leptin (R2=0.41, $P<0.0001)$ : omega-3 PUFA treatment and waist circumference (Table 2). In turn, independent predictors of $\mathrm{A} / \mathrm{L}$ ratio changes $(\mathrm{R} 2=0.53, P<0.0001)$ were: the assigned treatment, current smoking and hyperlipidemia (Table 2).

\section{Discussion}

This study demonstrates that omega-3 PUFA (eicosapentaenoic and docosahexaenoic acids) added to optimal medical and interventional therapy in patients with stable CAD favourably alter the plasma adipokine profile. We found that after 1-month treatment with omega-3 PUFA plasma adiponectin increased, while leptin decreased. This novel observation suggests an additional beneficial effect of omega-3 PUFA on cardiovascular risk and this effect is potent enough to be seen in the advanced CAD patients with several risk factors receiving
Table 2 Mulivariate analysis with adiponectin, leptin and adiponectin-to-leptin ratio changes during follow-up as the dependent variable
$A$ adiponectin, $L$ leptin, $1 M$

1 month, $B$ baseline

Adiponectin level change $=$

$\left(\mathrm{A}_{1 \mathrm{M}}-\mathrm{A}_{\mathrm{B}}\right) / \mathrm{A}_{\mathrm{B}} \times 100 \%$,

Leptin level change $=$

$\left(\mathrm{L}_{1 \mathrm{M}}-\mathrm{L}_{\mathrm{B}}\right) / \mathrm{L}_{\mathrm{B}} \times 100 \%$,

Adiponectin - to - leptin

ratio $=\left(\mathrm{A}_{1 \mathrm{M}} / \mathrm{L}_{1 \mathrm{M}}-\mathrm{A}_{\mathrm{B}} / \mathrm{L}_{\mathrm{B}}\right) /$

$\mathrm{A}_{\mathrm{B}} / \mathrm{L}_{\mathrm{B}} \cdot \times 100 \%$.

\begin{tabular}{llccc}
\hline Dependent variable & Independent variables & Coefficient & \% of variance & P-value \\
\hline Adiponectin level change & Treatment assignment & 0.61 & 27.5 & 0.0002 \\
R2=0.62, & Platelet level at baseline & -0.40 & 14.2 & 0.007 \\
F (6.25)=5.95, & Total cholesterol 1 month & 0.34 & 8.5 & 0.025 \\
$P<0.001$ & Current smoking & 0.30 & 6.1 & 0.05 \\
& Previous myocardial infarction & -0.19 & 3.1 & 0.19 \\
& Waist circumference & 0.04 & 0.01 & 0.77 \\
Leptin level change & Treatment assignment & -0.49 & 24.6 & 0.0002 \\
R2=0.41, & Waist circumference & -0.25 & 5.6 & 0.043 \\
F (3.44)=10.16, $P<0.0001$ & Hyperlipidemia & 0.19 & 2.8 & 0.11 \\
Adiponectin-to-leptin ratio & Treatment assignment & 0.51 & 25.2 & $<0.0001$ \\
R2=0.53, & Current smoking & 0.28 & 7.5 & 0.021 \\
F (6.41)=7.82, $P<0.0001$ & Hyperlipidemia & -0.24 & 6.5 & 0.037 \\
& Age & -0.05 & 0.2 & 0.648 \\
& Waist circumference & 0.16 & 2.5 & 0.159 \\
& Previous myocardial infarction & -0.17 & 1.9 & 0.142 \\
\hline
\end{tabular}


guideline-adjusted treatment, including statins, known to affect adipokines [17].

A drug-induced increase in circulating adiponectin, known to have cardioprotective effects [9], together with the decrease in leptin, are the major findings of the present study. Hypoadiponectinemia seems to be associated with inflammatory cardiovascular diseases, including aortic stenosis [18]. Several studies in animal models showed the potential of omega-3 PUFA to increase adiponectin in the circulating blood [19]. There are few studies on omega-3 PUFA-induced effects on adiponectin in humans. An increase in adiponectin was noticed after intervention with various doses of omega-3 in obese subjects [20]. On the other hand, no influence of omega3 PUFA on adiponectin was observed in healthy overweight to moderately obese men and women [21]. Mizia-Stec et al. demonstrated that adiponectin increased independently of omega-3 PUFA supplementation during the first month after acute myocardial infarction (AMI) [2], which may be explained by the recovery of AMI-induced acute phase pathophysiological abnormalities. Moreover, in the current study leptin decreased after intervention with omega-3 PUFA. Multiple studies regarding the effect of omega-3 PUFA on leptin, most of which performed in animal models, yielded conflicting results [15]. Puglisi et al. have recently suggested that omega-3 PUFA may downregulate leptin in association with reduced adiposity or upregulate its level in association with increased adiposity [22], whereas Sneddon et al. did not observe any change in leptin during administration of omega-3 PUFA in lean and obese men [23]. Our findings indicate that in $C A D$ patients omega-3 PUFA can significantly reduce leptin as early as after 30 days of therapy. To our knowledge, our study is the first to demonstrate increased adiponectin and reduced leptin in response to omega-3 PUFA treatment in stable CAD patients undergoing PCI, which could be clinically relevant.

Omega-3 PUFA-induced effects on resistin have attracted less interest. The potential of omega-3 PUFA to downregulate resistin gene expression has been shown in vitro [24]. In a small study [2] omega-3 PUFA in the first month after AMI contributed to a borderline increase in resistin. The discrepancy between that and our study, where no effect of omega-3 PUFA on resistin was observed, might be explained by a different study population (stable CAD versus AMI).

Omega-3 PUFA may incorporate into the membrane phospholipid fraction and TG lipid droplets of adipocytes, which might represent one of the mechanisms of their favorable effects [22]. They have been suggested to increase adiponectin secretion by peroxisome proliferator-activated receptorsgamma (PPAR-gamma) stimulation, the expression of which in adipose tissue is much greater than in other tissues [22]. This effect was completely blocked by administration of a PPAR-gamma inhibitor [25]. The role of this mechanism in regulating leptin is not clear, but there have been studies showing that a thiazolidinedione compound, a ligand for
$\operatorname{PPAR} \gamma$, decreased plasma leptin [26]. Thus it may be speculated that stimulation of PPAR $\gamma$ is one of the mechanisms by which omega-3 PUFA mediate their insulin sensitizing, lipid lowering, and anti-inflammatory properties.

Because adiponectin and leptin are associated with opposite metabolic effects, the $\mathrm{A} / \mathrm{L}$ ratio is a valuable variable. Some studies have reported that the $\mathrm{A} / \mathrm{L}$ ratio may serve as a reliable marker of insulin resistance [27]. It has also been found that the leptin to adiponectin ratio positively correlated with IMT [28]. We found that omega-3 PUFA can increase that ratio in CAD patients, which is a novel observation, corroborating a beneficial effect of this agent on the adipokine profile.

Despite multiple beneficial effects of omega-3 PUFA on the cardiovascular system [1-4], the recently published studies have shown that omega-3 PUFA supplementation was not associated with a lower risk of MACE in patients at increased cardiovascular risk [29]. It may be speculated that the current guideline-adjusted treatment of CAD interferes with omega-3 PUFA-induced cardioprotective effects, which might be abolished by high-dose statins and angiotensin converting enzyme inhibitors (ACE-I) intake. Results of our recent studies $[3,4]$ in such patients have demonstrated that the effect of omega-3 PUFA at a dose of $1 \mathrm{~g} / \mathrm{d}$ on biomarkers of platelet activation, coagulation and oxidative stress is moderate. Therefore, it might be hypothesized that a higher dose of omega-3 PUFA on top of optimal medical therapy is required to exert meaningful clinical effects.

The study has several limitations. Firstly, the sample size is relatively small. However, although the study was randomized, both groups were well matched and represented typical stable angina patients undergoing PCI. Another shortcoming is a relatively short follow-up. Therefore we could not evaluate clinical end points to establish whether the effects of omega-3 PUFA on adipokine profile improved clinical outcomes. Finally, since the duration of the study was too limited to expect significant changes in body weight and waist circumference we did not measure adipose tissue directly. However, alterations in adipose tissue mass and/or morphology cannot be eliminated.

In conclusion, we have demonstrated in high risk stable CAD patients who underwent PCI that omega-3 PUFA supplementation improves adipokine profile. This might be a novel, favorable mechanism of omega-3 PUFA action.

Open Access This article is distributed under the terms of the Creative Commons Attribution License which permits any use, distribution, and reproduction in any medium, provided the original author(s) and the source are credited.

\section{References}

1. Kar S, Webel R. Fish oil supplementation \& coronary artery disease: does it help? Mo Med. 2012;109:142-5. 
2. Mizia-Stec K, Haberka M, Mizia M, et al. N-3 Polyunsaturated fatty acid therapy improves endothelial function and affects adiponectin and resistin balance in the first month after myocardial infarction. Arch Med Sci. 2011;7:788-95.

3. Gajos G, Rostoff P, Undas A, et al. Effect of polyunsaturated omega-3 fatty acids on responsiveness to dual antiplatelet therapy in patients undergoing percutaneus coronary intervention: the OMEGA-PCI study. J Am Coll Cardiol. 2010;55:1671-8.

4. Gajos G, Zalewski J, Rostoff P, et al. Reduced thrombin formation and altered fibrin clot properties induced by polyunsaturated omega-3 fatty acids on top of dual antiplatelet therapy in patients undergoing percutaneus coronary intervention (OMEGA-PCI clot.). Arterioscler Thromb Vasc Biol. 2011;31:1696-702.

5. European Guidelines on cardiovascular disease prevention in clinical practice (version 2012). The fifth joint task force of the european society of cardiology and other societies on cardiovascular disease prevention in clinical practice. Eur Heart J. 2012;33:1635-701.

6. Kromhout D, Giltay EJ, Geleijnse JM. Alpha Omega Trial Group. n-3 fatty acids and cardiovascular events after myocardial infarction. N Engl J Med. 2010;363:2015-26.

7. Chaldakov GN, Stankulov IS, Hristova M, Ghenev PI. Adipobiology of disease: adipokines and adipokine-targeted pharmacology. Curr Pharm Des. 2003;9:1023-31.

8. West of Scotland Coronary Prevention Study: identification of high-risk groups and comparison with other cardiovascular intervention trials. Lancet. 1996; 348(9038):1339-42.

9. Shioji K, Moriwaki S, Takeuchi Y, Uegaito T, Mutsuo S, Matsuda M. Relationship of serum adiponectin level to adverse cardiovascular events in patients who undergo percutaneous coronary intervention. Circ J. 2007;71:675-80.

10. Nogueiras R, Novelle MG, Vazquez MJ, Lopez M, Dieguez C. Resistin: regulation of food intake, glucose homeostasis and lipid metabolism. Endocr Dev. 2010;17:175-84.

11. Masquio DC, de Piano A, Sanches PL. The effect of weight loss magnitude on pro/anti-inflammatory adipokines and carotid intima-media thickness in obese adolescents engaged in interdisciplinary weight-loss therapy. Clin Endocrinol (Oxf). 2012 [Epub ahead of print].

12. Sassen LM, Lamers JM, Sluiter W, et al. Development and regression of atherosclerosis in pigs. Effects of $n-3$ fatty acids, their incorporation into plasma and aortic plaque lipids, and granulocyte function. Arterioscler Thromb. 1993;13:651-60.

13. Guallar E, Aro A, Jiménez FJ, et al. Omega-3 fatty acids in adipose tissue and risk of myocardial infarction: the EURAMIC study. Arterioscler Thromb Vasc Biol. 1999;19:1111-8.

14. Moreno-Aliaga MJ, Lorente-Cebrián S, Martínez JA. Regulation of adipokine secretion by n-3 fatty acids. Proc Nutr Soc. 2010;69:324-32.

15. Wanders D, Plaisance EP, Judd RL. Pharmacological effects of lipid-lowering drugs on circulating adipokines. World J Diabetes. 2010;1:116-28.

16. Alberti KG, Eckel RH, Grundy SM, et al. Harmonizing the metabolic syndrome: A Joint Interim Statement of the International Diabetes Federation Task Force on Epidemiology and
Prevention; National Heart, Lung, and Blood Institute; American Heart Association; World Heart Federation; International Atherosclerosis Society; and International Association for the Study of Obesity. Circulation. 2009;120:1640-5.

17. Sakamoto K, Sakamoto T, Ogawa H, Kurnamoto Joint Research on Hypercholesterolemia Investigators. The effect of 6 months of treatment with pravastatin on serum adiponection concentrations in Japanese patients with coronary artery disease and hypercholesterolemia: a pilot study. Clin Ther. 2006;28:1012-21.

18. Kolasa-Trela R, Miszalski-Jamka T, Grudzień G, Wypasek E, Kostkiewicz M. Adiponectin, leptin, and resistin in patients with aortic stenosis without concomitant atherosclerotic vascular disease. Pol Arch Med Wewn. 2011;121:352-9.

19. Rossi AS, Lombardo YB, Lacorte JM, et al. Dietary fish oil positively regulates plasma leptin and adiponectin levels in sucrose-fed, insulin-resistant rats. Am J Physiol Regul Integr Comp Physiol. 2005;289:486-94.

20. Itoh M, Suganami T, Satoh N, et al. Increased adiponectin secretion by highly purified eicosapentaenoic acid in rodent models of obesity and human obese subjects. Arterioscler Thromb Vasc Biol. 2007;27:1918-25.

21. Kratz M, Swarbrick MM, Callahan HS, Matthys CC, Havel PJ, Weigle DS. Effect of dietary n-3 polyunsaturated fatty acids on plasma total and high-molecular-weight adiponectin concentrations in overweight to moderately obese men and women. Am J Clin Nutr. 2008;87:347-53.

22. Puglisi MJ, Hasty AH, Saraswathi V. The role of adipose tissue in mediating the beneficial effects of dietary fish oil. J Nutr Biochem. 2011;22:101-8

23. Sneddon AA, Tsofliou F, Fyfe CL, et al. Effect of a conjugated linoleic acid and omega-3 fatty acid mixture on body composition and adiponectin. Obesity. 2008;16:1019-24.

24. Haugen F, Zahid N, Dalen KT, Hollung K, Nebb HI, Drevon CA. Resistin expression in 3T3-L1 adipocytes is reduced by arachidonic acid. J Lipid Res. 2005;46:143-53.

25. Neschen S, Morino K, Rossbacher JC. Fish oil regulates adiponectin secretion by a peroxisome proliferator-activated receptor-gammadependent mechanism in mice. Diabetes. 2006;55:924-8.

26. Kallen CB, Lazar MA. Antidiabetic thiazolidinediones inhibit leptin (ob) gene expression in 3T3-L1 adipocytes. Proc Natl Acad Sci USA. 1996;93:5793-6.

27. Zaletel J, Barlovic DP, Prezelj J. Adiponectin-leptin ratio: a useful estimate of insulin resistance in patients with Type 2 diabetes. $\mathrm{J}$ Endocrinol Invest. 2010;33:514-8.

28. Kotani K, Sakane N, Saiga K, Kurozawa Y. Leptin:adiponectin ratio as an atherosclerotic index in patients with type 2 diabetes: relationship of the index to carotid intima-media thickness. Diabetologia. 2005;48:2684-6.

29. Rizos EC, Ntzani EE, Bika E, Kostapanos MS, Elisaf MS. Association between omega-3 fatty acid supplementation and risk of major cardiovascular disease events: a systematic review and meta-analysis. JAMA. 2012;308:1024-33. 